\title{
Should point-of-care ultrasound become part of healthcare worker testing for COVID?
}

\author{
Authors: Nicholas Smallwood, ${ }^{A}$ Andrew Walden, ${ }^{B}$ Prashant Parulekar ${ }^{\mathrm{C}}$ and Martin Dachse ${ }^{\mathrm{A}}$
}

\begin{abstract}
The NHS in England has rapidly expanded staff testing for COVID-19 in order to allow healthcare workers who would otherwise be isolating with symptoms suspicious of COVID-19 to be cleared to work. However, the high false negative rate associated with current RT-PCR tests could put other staff, family members and patients at risk. We believe combining swab testing with real-time lung ultrasound (LUS) would improve the ability to rule-in COVID-19 infection in those requiring screening.
\end{abstract}

KEYWORDS: POCUS, screening, COVID-19

DOI:10.7861/clinmed.2020-0442

\section{Background}

The NHS in England has rapidly expanded staff testing for COVID-19 in order to reduce the burden of healthcare worker (HCW) absence on NHS resources. This test uses RT-PCR to detect viral RNA and has a quoted sensitivity of approximately $60-70 \%$. This means that three to four out of 10 healthcare workers with suspicion of COVID-19 infection may receive a false negative result and be cleared to work while still infectious. This raises the question of the reliability of a SARS-CoV-2 swab in isolation as a means of allowing staff to safely return to work, and thereby put other staff, family members and patients at risk.

\section{Combining lung ultrasound with swab testing to improve test sensitivity}

We believe combining swab testing with real-time lung ultrasound (LUS) would improve the ability to rule-in COVID-19 infection in those requiring screening. The sonographic appearances of interstitial pneumonitis have been well-described in COVID-19 patients, ${ }^{2,3}$ and numerous anecdotes and case series ${ }^{4}$ demonstrate interstitial or 'ground glass' changes on CT even when patients are asymptomatic. We also know that lung ultrasound approximates $\mathrm{CT}$ in diagnostic accuracy when visualising lung parenchyma

Authors: Aconsultant in acute medicine, East Surrey Hospital, Redhill, UK; ${ }^{\mathrm{B}}$ consultant in acute and intensive care medicine, Royal Berkshire Hospital, Reading, UK; ' ${ }^{C}$ consultant in acute and intensive care medicine, William Harvey Hospital, Ashford, UK in patients with respiratory failure, ${ }^{5}$ and perhaps is even more sensitive than CT in the diagnosis of COVID-19. ${ }^{6}$ Therefore, LUS may be expected to pick up changes related to COVID-19 even in asymptomatic or mildly symptomatic patients, adding diagnostic accuracy to the RT-PCR screening process.

This is most likely to be true in those HCWs without pre-existing pleural or fibrotic lung disease, in whom normal lung ultrasound appearances would be expected. Any deviation from a normal scan in the presence of clinical features requiring screening would, we suggest, be highly suspicious for COVID-19 regardless of the result of RT-PCR testing. Conversely, a negative LUS showing normal appearances would be reassuring, but would still require a negative RT-PCR result before the HCW would be considered suitable for work. We do not know the true incidence of COVID 19 infection without radiological pneumonitis, although it may well exist.

Using this combined approach, the sensitivity of screening is likely to be increased, since there would be some workers with infection found through LUS that would otherwise be missed through a single RT-PCR swab alone. We would propose a positive LUS but negative RT-PCR should prompt re-swab and continued absence from work of the HCW. This does have the potential to lead to a small increase in false positives (and therefore reduced specificity of screening), but we consider it highly unlikely that $\mathrm{HCW}$ s without prior lung disease and/or active COVID infection would return a positive LUS result. In such a scenario, we would suggest the risks of a false positive result delaying the HCW returning to work for a couple of days while awaiting a repeat swab are much less than the demonstrable risks of infected HCWs inadvertently returning to work while infectious due to a false negative swab result.

Combined screening would not delay those HCWs who do not have evidence of infection being able to go back to work, since the LUS results will be available immediately and if negative, the worker would remain in isolation until a negative swab result is also returned. Therefore, this would not prolong the current screening process for true negative HCWs. For the anticipated small number of false positive HCWs, combined screening would introduce a delay of a couple of days while awaiting a repeat swab result. We believe in the absence of significant symptoms, two negative RT-PCR results would indicate a very low probability of infection, even with an abnormal lung ultrasound (although there is no data available on the exact sensitivity of multiple swabs in asymptomatic or minimally symptomatic patients, as far as we are aware). The delays associated will continue to fall as the processing time of RT-PCR swabs continues to decrease. 


\section{Lung ultrasound without swab testing for regular screening}

A further option to testing asymptomatic (or minimally symptomatic) HCWs would be to use LUS as part of a regular screening programme for certain groups of asymptomatic HCWs without pre-existing lung disease. This could be of particular relevance in areas where asymptomatic transmission to patients, staff or families carries a particularly high risk (for example those from the BAME community, the immunosuppressed, critically ill or care home residents). Since the pre-test probability in an asymptomatic HCW is low to moderate, LUS as a test with high sensitivity should significantly reduce the incidence of asymptomatic transmission from HCWs, reassuring staff, families and patients.

\section{Validation}

It must be highlighted that there is currently no clear data to support the use of LUS as a screening tool, since there is to our knowledge no published data on the utility of LUS in asymptomatic or minimally symptomatic patients (ie those not requiring medical assessment). There is small volume data published establishing the utility of LUS in the diagnosis of those admitted with COVID infection, ${ }^{6}$ and larger trial data have been submitted but not yet accepted for publication (N Smallwood, personal communication). Despite this relative lack of data, we believe the risks are minimal, and the potential benefits significant: LUS is quick (it takes less than 5 minutes for a 12-point scan), noninvasive, and if stringent infection control measures are applied there should be minimal risk of cross-contamination.

Of course, the only way to entirely validate a dual approach to testing such as this is to conduct a research trial, in which the gold standard for the exclusion of COVID-19 would have to be clearly defined. This is likely to require multiple RT-PCR swabs, since the sensitivity of testing increases with multiple swabs, and perhaps delayed testing of COVID-19 antibodies as an additional data point. This would allow the generation of an evidence-based risk-prediction tool for use as part of the screening process. Any research would not require randomisation or control since the data required is to ascertain the sensitivity and specificity of LUS for the diagnosis of COVID-19 in asymptomatic or minimally symptomatic (but at risk) individuals. Another option would be to compare LUS to low dose CT scan as the imaging of choice for screening patients, and as mentioned there are already trials underway to compare the accuracy of LUS versus CT scan in those admitted with COVID-19 infection.

\section{Overcoming barriers to implementation}

One potential issue we acknowledge is that it is not clear if such a proposal could be implemented on a widespread basis, since there are well established barriers to national uptake of point of care ultrasound. ${ }^{7}$ But even if only some centres were able to implement this practice to the benefit of their staff and patients, we feel there is a clear rationale to consider this approach. The numbers of accredited practitioners in both focused acute medicine ultrasound (FAMUS $)^{8}$ and focused ultrasound in intensive care $(\text { FUSIC })^{9}$ - both of which include LUS at their core - is steadily increasing. This means that if such an approach were proven worthwhile, the number of sites able to deliver this additional testing would be increasing all the time. The cost implications of on-site testing would not be expected to be significant since the majority of sites with LUS-trained clinicians are expected to have access to ultrasound machines. If screening were occurring off-site, however, then appropriate hardware (and staff time) would have to be factored in. High quality, portable ultrasound machines are now available for approximately $£ 2,000$ and there are groups that are defining pathways to allow the rapid training of non-hospital clinicians in LUS for the diagnosis of COVID-19 (for example the TICC-19 pathway; https://ticc19.com/).

\section{Conclusion}

We propose this combination of non-ionising imaging with RT-PCR testing should be incorporated into clinical practice in those organisations where there is the ability to provide it, pending further research into its utility to improve the accuracy of screening for HCWs for COVID-19. In due course, we will be able to ascertain whether PCR or LUS alone, PCR with LUS or perhaps even PCR with low dose CT are the safest, most accurate and cost-effective testing regime in a number of clinical scenarios. The benefits of a combined LUS approach seem entirely plausible, while the risks of continuing with an isolated screening test with a significant false negative rate should not be ignored.

\section{Conflicts of interest}

Andrew Walden is the director of Box Courses which runs point-ofcare ultrasound courses.

\section{References}

1 Wang W, Xu Y, Gao R et al. Detection of SARS-CoV-2 in different types of clinical specimens. JAMA 2020;323:1843-4.

2 Peng Q-Y, Wang X-T, Zhang L-N, Chinese Critical Care Ultrasound Study Group (CCUSG). Findings of lung ultrasonography of novel corona virus pneumonia during the 2019-2020 epidemic. Intensive Care Med 2020;46:849-50.

3 Volpicelli G, Lamorte A, Villén T. What's new in lung ultrasound during the COVID-19 pandemic. Intensive Care Med 2020;46:1445-8.

4 Albano D, Bertagna F, Bertolia $M$ et al. Incidental findings suggestive of COVID-19 in asymptomatic patients undergoing nuclear medicine procedures in a high prevalence region. J Nucl Med 2020;61:632-6.

5 Tierney DM, Huelster JS, Overgaard JD et al. Comparative performance of pulmonary ultrasound, chest radiograph, and CT among patients with acute respiratory failure. Crit Care Med 2020;48:151-7.

6 Yang Y, Huang Y, Gao F, Yuan L, Wang Z. Lung ultrasonography versus chest CT in COVID-19 pneumonia: a two-centered retrospective comparison study from China. Intensive Care Med 2020, in press (doi: 10.1007/s00134-020-06096-1).

7 Smallwood N, Matsa R, Lawrenson P, Messenger J, Walden A. A UK wide survey on attitudes to point of care ultrasound training amongst clinicians working on the Acute Medical Unit. Acute Med 2016;14:159-64.

8 Smallwood N, Dachsel M, Matsa R et al. Focused acute medicine ultrasound (FAMUS) - point of care ultrasound for the acute medical unit. Acute Med 2016;15:193-6.

9 Intensive Care Society. Focused ultrasound for intensive care. www. ics.ac.uk/ICS/FUSIC/ICS/FUSIC/FUSIC_Accreditation.aspx?hkey= c88fa5cd-5c3f-4c22-b007-53e01a523ce8 [Accessed 8 June 2020].

Address for correspondence: Dr Nicholas Smallwood, Department of Acute Medicine, East Surrey Hospital, Canada Avenue, Redhill RH1 5RH, UK.

Email: nicholas.smallwood@nhs.net 